\title{
SIMULACIÓN DE LA FENOLOGÍA, CRECIMIENTO Y RENDIMIENTO SIN LIMITACIONES HÍDRICAS DE LA VARIEDAD ASEM 400 INTA DE MANÍ CON DSSAT CROP PEANUT MODEL
}

\author{
Gustavo Ovando ${ }^{1}$ e Ricardo Javier Haro ${ }^{2}$
}

${ }^{1}$ Facultad de Ciencias Agropecuarias, Universidad Nacional de Córdoba, Argentina; ${ }^{2}$ INTA-EEA Manfredi, Córdoba, Argentina

RESUMEN: DSSAT es una herramienta valiosa para la toma de decisiones sobre el manejo del cultivo pero previamente debe ser calibrado y validado según genotipos y ambientes. El objetivo de este trabajo fue calibrar los coeficientes genéticos del modelo CROPGRO-Peanut asociados a fenología, producción de biomasa y rendimiento para el cultivar ASEM 400 INTA. El modelo generó simulaciones con valores similares a los observados a campo. La predicción a eventos fenológicos sólo manifestó leve inexactitud (2 días) sobre inicio de crecimiento de vainas. La simulación de producción de biomasa total y de vainas fue semejante a valores observados $(d \geq 0.98) \mathrm{y}$, aquellos valores simulados de rendimiento y sus componentes principales, relación grano-caja e índice de cosecha de vainas fueron similares a los observados. Los resultados de la calibración avalan la precisión de DSSAT para predecir la fenología y rasgos asociados al crecimiento del cultivar ASEM 400 INTA.

Palabras-Clave: Modelo de cultivo, coeficiente genético, calibración, productividad.

\section{INTRODUCCIÓN}

La provincia de Córdoba es la principal productora de maní (Arachis hypogaea L.) de Argentina. En la campaña 2017/18 se sembraron 498 mil hectáreas de maní de las cuales el 90\% correspondió a la superficie cultivada por Córdoba, constituyéndose ésta en una economía regional de importancia destacada (BARBERIS et al., 2018).

En un mundo en donde la población está constantemente creciendo, resulta de vital importancia monitorear la producción de los cultivos y pronosticar los rendimientos a los efectos de garantizar la seguridad alimentaria. Por lo tanto, es necesario tener herramientas que permitan estimar el rendimiento de los cultivos sobre grandes áreas (BATTUDE et al., 2016) particularmente en las regiones áridas y semiáridas (CHAHBIBELLAKANJI et al., 2018). En tal sentido varios modelos de cultivos basados en procesos han sido desarrollados para estimar la producción de los cultivos (BATTUDE et al., 2016). 


\section{Encontro Sobre a Cultura do Amendoim \\ 15 a 17 de agosto de 2019 na Estação de Eventos Cora Coralina e FCAV/UNESP, Jaboticabal-SP}

Los modelos de cultivo Decision Support System for Agrotechnology Transfer (DSSAT) ha sido ampliamente usado para diferentes aplicaciones en todo el mundo. DSSAT es una herramienta valiosa para la toma de decisiones sobre el manejo del cultivo al reducir los tiempos y costos requeridos para analizar decisiones alternativas complejas y también provee un entorno de trabajo para la cooperación científica al integrar nuevos conocimientos y aplicarlos a diferentes temas de investigación (MFWANGO et al., 2018). El modelo CROPGRO-Peanut es una estructura modular del DSSAT que simula la fenología y productividad del cultivo de maní bajo condiciones ambientales y prácticas de manejo. Sin embargo, el modelo requiere no sólo información de suelo, clima y prácticas de manejo; pues también demanda información sobre coeficientes genéticos específicos de cada cultivar que definen el desarrollo y el crecimiento. Para que pueda predecir adecuadamente el modelo requiere, ser calibrado en primera instancia y más tarde validado (HARO y OVANDO, 2016a).

El objetivo de este trabajo fue calibrar los coeficientes genéticos del modelo CROPGROPeanut asociados a fenología, producción de biomasa y rendimiento para el cultivar ASEM 400 INTA.

\section{MATERIALES Y MÉTODOS}

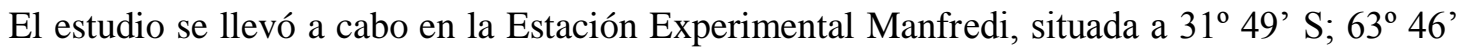
O y 292 msnm, en un área geomorfológica que se conoce con el nombre de Pampa alta o elevada. El cultivar ASEM 400 INTA fue sembrado en campo el 30 de octubre de 2017, el mismo creció sin restricción hídrica y fue manejado según recomendaciones habituales para esta especie. Se realizaron muestreos de biomasa a partir de floración y continuaron periódicamente cada 15 días hasta cosecha. En cada fecha de muestreo se recolectaron plantas sobre una superficie de $0,70 \mathrm{~m}^{2} \mathrm{y}$, la biomasa fue separada en hojas y tallo (biomasa vegetativa) y vainas (biomasa reproductiva) para luego ser secadas. A cosecha, las vainas y los granos fueron pesados para la obtención del rendimiento, el número y peso de granos, la relación granos/caja e índice de cosecha de vainas.

Para simular el cultivar ASEM 400INTA mediante el modelo CROPGRO-Peanut del paquete DSSAT versión 4.6 se tomó como base los coeficientes genéticos de la variedad ASEM 485 INTA (HARO y OVANDO, 2016b). Luego, los coeficientes genéticos relacionados a la fenología, producción de biomasa y rendimiento fueron modificados. La calibración fue valorada mediante gráficos de variables que incluyeron valores observados (a campo) y valores estimados (por el modelo) y, mediante los estadísticos: coeficiente de concordancia de Willmott (d), error medio (MBE) y la raíz del error cuadrático medio (RMSE).

\section{RESULTADOS Y DISCUSIÓN}

Los valores de coeficientes genéticos del cultivar ASEM 400 INTA, determinados luego de la calibración se muestran en Tabla 1. A partir de los coeficientes genéticos asociados a fenología se simuló la duración de diversos subperíodos fenológicos y se comparó con los valores observados, 


\section{Encontro Sobre a Cultura do Amendoim \\ 15 a 17 de agosto de 2019 na Estação de Eventos Cora Coralina e FCAV/UNESP, Jaboticabal-SP}

siendo las duraciones simuladas desde siembra a: floración (R1), inicio de crecimientos de vaina (R3) y de grano (R5) de 39, 64 y 74 días, respectivamente; mientras que los valores observados para los mismos subperíodos fueron de 39; 62 y 74 días, respectivamente, quedando plasmado un buen ajuste entre valores simulados y estimados en lo referente a fenología. Los valores de los coeficientes genéticos para fenología de ASEM 400 INTA reflejan el acortamiento de ciclo de este cultivar, habitualmente manifestado a campo, respecto de ASEM 485 INTA (genotipo proveedor de los coeficientes genéticos iniciales). Dos aspectos de ASEM 400 INTA merecen destacarse: (i) precoz emergencia de plantas que es traducida en pronto establecimiento poblacional a campo y, (ii) acortamiento del período de llenado de grano acompañado de una marcada partición de fotoasimilados hacia dichas estructuras reproductivas.

Tabla 1. Coeficientes genéticos del cultivar ASEM 400 INTA para subperíodos fenológicos, determinación del número de semillas por vaina, peso de la semilla y composición de aceite del grano.

\section{Coeficiente}

\section{Genético}

\section{Período o Rasgo}

\section{Fenología}

17,0 EM-FL: Tiempo (Días fototermales) entre la emergencia de la planta y primera flor $(\mathrm{R} 1)$.

13,0 FL-SH: Tiempo (Días fototermales) entre primera flor y primera vaina (R3).

25,7 FL-SD: Tiempo (Días fototermales) entre primera flor y primera semilla (R5).

27,0 SD-PM: Tiempo (Días fototermales) entre primera semilla y

Crecimiento madurez fisiológica (R7)

0,667 WTPSD: Peso máximo de cada semilla (g)

26,5 SFDUR: Duración del llenado de las semilla para cada cohorte de vainas (Días fototermales).

1,83 SDPDV: Número promedio de semillas por vaina.

14,0 PODUR: Tiempo (Días fototermales) requerido por la variedad para completar el llenado de la vaina bajo condiciones óptimas.

0,470 SDLIP: Fracción de aceite en las semillas (g(aceite) $/ \mathrm{g}$ (semilla)

Las estimaciones de la biomasa total y vainas por el modelo CROPGRO-Peanut ajustaron a funciones de tipo sigmoidea con valores similares a aquellos observados a campo (Fig. 1). Los estadísticos para biomasa total y de vaina (Tabla 2) resultan concordantes con los aquellos determinados por otros autores (ANOTHAI et al., 2008; Guerra et al., 2008), quienes evaluaron cultivares de rasgos de canopeo similares al de ASEM 400 INTA. El modelo subestimó el peso seco de vainas, lo cual es explicitado mediante signo negativo de MBE, y dicha respuesta coincide con lo determinado por Yadav et al. (2012). Sin embargo, el ajuste entre observado y simulado a cosecha fue preciso (observado: $6384 \mathrm{~kg} / \mathrm{ha}$; simulado: $6307 \mathrm{~kg} / \mathrm{ha}$ ). 


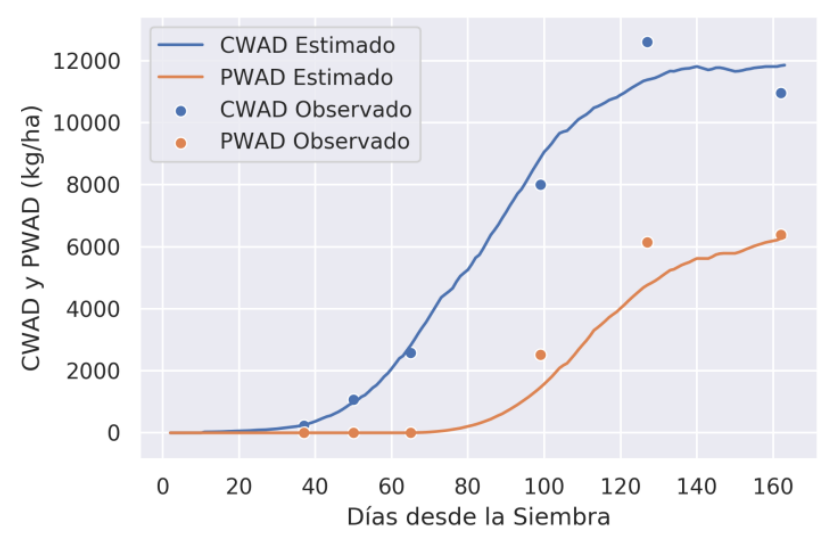

Figura 1. Valores observados (círculos) y estimados (líneas) de biomasa total (CWAD, kg/ha) y de vainas (PWAD, kg/ha) a lo largo del ciclo del cultivar ASEM 400 INTA.

Tabla 2. Errores de la estimación de producción de biomasa.

\begin{tabular}{ccc}
\hline & CWAD & PWAD \\
\hline$d$ & 0,99 & 0,98 \\
$\operatorname{RMSE}(\mathrm{kg} / \mathrm{ha})$ & 662 & 656 \\
MBE $(\mathrm{kg} / \mathrm{ha})$ & 95 & -364 \\
\hline
\end{tabular}

El rendimiento en granos simulado fue preciso (observado y estimado de $5057 \mathrm{~kg} / \mathrm{ha}$ y $5101 \mathrm{~kg} / \mathrm{ha}$, respectivamente), en consonancia con la semejanza entre observados y simulados del número de granos y peso del grano como representantes de los componentes principales del rendimiento. El número de granos fue 809 granos $/ \mathrm{m}^{2}$ y 807 granos $/ \mathrm{m}^{2}$ para observados y simulados, respectivamente; en tanto que, el peso de grano fue $625,1 \mathrm{mg} /$ grano y $632,5 \mathrm{mg} / \mathrm{grano}$ para observados y simulados, respectivamente. Las similitudes entre valores observados y simulados de número de granos y peso de grano son evidencias conclusiva y robusta de la óptima calibración de biomasa total y de vainas y, de la partición implícita de fotoasimilados hacia estructuras reproductivas a través del ciclo del cultivo. La relación granos/caja e índice de cosecha de vainas son rasgos que también expresan la partición hacia órganos reproductivos y, por ende, una modelización precisa de estos rasgos es de gran importancia. La relación grano/caja fue $79 \%$ y $81 \%$ para observados y simulados, respectivamente; en tanto que, el índice de cosecha de vainas fue 0,58 y 0,53 para observados y simulados, respectivamente.

\section{CONCLUSIONES}

El modelo DSSAT CROP PEANUT, una vez calibrado, permitió estimar adecuadamente la fenología, crecimiento y el rendimiento de la variedad ASEM 400 INTA. 


\section{Encontro Sobre a Cultura do Amendoim}

15 a 17 de agosto de 2019 na Estação de Eventos Cora Coralina e FCAV/UNESP, Jaboticabal-SP

La calibración bajo la condición riego es una primera aproximación importante, pero dichos coeficientes genéticos deben ser validados en condiciones de secano para la región en estudio.

\section{AGRADECIMENTOS}

La investigación fue financiada por INTA y Fundación Maní Argentino.

\section{REFERENCIAS BIBLIOGRÁFICAS}

ANOTHAI, J.; PATANOTHAI, A.; JOGLOY, S.; PANNANGPETCH, K.; BOOTE, K. J.; HOOGENBOOM, G. A sequential approach for determining the cultivar coefficients of peanut lines using end-of-season data of crop performance trials. Field Crops Research, v.108 (2), p. 169-178, 2008.

BARBERIS, N.; BONGIOVANNI, R.; GILETTA, M. A. Resultado económico esperado de la agricultura, campaña agrícola 2018/19, Córdoba. Departamentos: Colón, Río Primero, Río Segundo y Tercero Arriba (noviembre 2018). EEA Manfredi, INTA, 2018.

BATTUDE, M.; AL BITAR, A.; MORIN, D.; CROS, J.; HUC, M., SICRE, C. M.; LE DANTEC, V.; DEMAREZ, V. Estimating maize biomass and yield over large areas using high spatial and temporal resolution Sentinel-2 like remote sensing data. Remote Sensing of Environment, V.184, p. 668-681, 2016.

CHAHBIBELLAKANJI, A.; ZRIBI, M.; LILI-CHABAANE, Z.; MOUGENOT, B. Forecasting of Cereal Yields in a Semi-arid Area Using the Simple Algorithm for Yield Estimation (SAFY) AgroMeteorological Model Combined with Optical SPOT/HRV Images. Sensors, v.18 (7), p.2138, 2018.

GUERRA, L. C.; HOOGENBOOM, G.; GARCIA Y GARCIA, A.; BANTERNG, P.; BEASLEY JR, J. P. Determination of cultivar coefficients for the CSM-CROPGRO-Peanut model using variety trial data. Trans. ASABE, v.51 (4), p.1471-1481, 2008.

HARO, R. J.; OVANDO, G. Modelo CROPGRO-Peanut: simulación de la fenología, crecimiento y rendimiento del cultivo de maní. XXXI Jornada Nacional de Maní en General Cabrera - Córdoba Argentina, 22 de septiembre, $2016 \mathrm{~b}$.

HARO, R.J.; OVANDO, G. Simulación de la fenología del cultivo de maní en Argentina mediante el modelo DSSAT. XIII Encontro sobre a cultura do Amendoim. Jaboticabal, San Pablo, Brasil. 18 y 19 de agosto de 2016a.

MFWANGO, L. H.; MFWANGO, L. H.; PRANUTHI, G.; DUBEY, S. K.; GUBEY, V. K. Application of Decision Support System for Agro Technology Transfer (DSSAT) to Simulate Agronomic Practices for Cultivation of Maize in Southern Highland of Tanzania. Agricultural Sciences, v.9 (07), 910, p. 2018. 
XVI Encontro Sobre a Cultura do Amendoim

15 a 17 de agosto de 2019 na Estação de Eventos Cora Coralina e FCAV/UNESP, Jaboticabal-SP

YADAV, S. B.; PATEL, H. R.; PATEL, G. G.; LUNAGARIA, M. M.; KARANDE, B. I.; SHAH, A. V.; PANDEY, V. Calibration and validation of PNUTGRO (DSSAT v4. 5) model for yield and yield attributing characters of kharif groundnut cultivars in middle Gujarat region. Journal of Agrometeorology, v.1, p. 24-29, 2012. 